\title{
THE ELECTRICAL RESISTIVITY OF MONOCRYSTALLINE FILMS DEDUCED FROM DERIVATION OF THE MAYADAS-SHATZKES EQUATIONS
}

\author{
C. R. TELLIER and A. J. TOSSER \\ Université de Nancy-I Laboratoire d'Electronique. Case Officielle 140 - 54037 NANCY Cedex - \\ FRANCE
}

(Received March 23, 1976; in final form August 29, 1976)

\begin{abstract}
The theoretical expression deduced by Mola and Heras from the Mayadas-Shatzkes model of monocrystalline thin film resistivity is approximated assuming that the Sommerfeld relation is valid and introducing corrections to the Matthiessen's rule.

The approximate expression of $\mathrm{k} \rho \mathrm{f} / \rho_{0}$ deviates from less than $6 \%$ in the $0.01-2 \mathrm{k}$-range, $0-0.5 p$-range and $0.1-0.62 r$-range.
\end{abstract}

\section{INTRODUCTION}

Mayadas and Shatzkes ${ }^{1}$ have proposed a theoretical calculation of the electrical resistivity of thin metallic films; they have considered the simultaneous action of isotropic electron scattering, surface scattering and grain boundary scattering. Mola and Heras ${ }^{2}$ have tabulated the exact values of the electrical resistivity of monocrystalline and polycrystalline metal films and given linearized expressions ${ }^{3}$ which are valid in some special cases $(\mathrm{p}=0,0.1 \leqslant \mathrm{k} \leqslant 5,0.1 \leqslant \mathrm{r} \leqslant 0.52)$. In this paper we propose an approximate expression of the resistivity of monocrystalline films which is valid in larger ranges.

\section{APPROXIMATE EXPRESSION OF MONOCRYSTALLINE FILM RESISTIVITY}

The resistivity $\rho_{\mathrm{f}}$ of a monocrystalline film is ${ }^{3}$ given by

$$
\rho_{\mathrm{f}} / \rho_{0}=[f(\alpha)-A]^{-1}
$$

in which $\rho_{0}$ is the resistivity of an infinitely thick monocrystalline film, i.e. without Fuchs-

Sondheimer ${ }^{4}$ size effects, where

$$
\begin{aligned}
A= & \frac{6}{\pi k}(1-p) \int_{0}^{\pi / 2} \int_{1}^{\infty} \frac{\cos ^{2} \phi}{H^{2}(t, \phi)}\left(\frac{1}{t^{3}}-\frac{1}{t^{5}}\right) \\
& \times \frac{1-\exp \{-k \cdot t \cdot H(t, \phi)\}}{1-p \exp \{-k \cdot t \cdot H(t, \phi)\}} \mathrm{d} t, \mathrm{~d} \phi
\end{aligned}
$$

and where

$$
f(\alpha)=1-\frac{3 \alpha}{2}+3 \alpha^{2}-3 \alpha^{3} \log _{\epsilon}\left(1+\frac{1}{\alpha}\right)
$$

The variable $p$ is the fraction of electrons specularly scattered at the external surfaces and $k$ is the ratio of film thickness, $d$, to electron mean free path, $l_{0}$.

$$
\begin{aligned}
k & =\mathrm{d} / l_{0} \\
\alpha & =(1 / k) . r .(1-r)^{-1}
\end{aligned}
$$

where $r$ is the electron reflection coefficient defined by the relation ${ }^{1}$

$$
r(1-r)^{-1}=\frac{1}{2}(V \cdot a)^{2} \cdot\left(\hbar^{2} k_{\mathbf{f}} / 2 m\right)^{-2}
$$

in which $V$ is the height of the potential well at grainboundary and $a$ is the potential well width, $\hbar$ is the modified Planck's constant, $k_{\mathrm{f}}$ the magnitude of the Fermi wave vector and $m$ the electron effective mass.

$$
H(t, \phi)=1+\alpha(\cos \phi)^{-1} \cdot\left(1-1 / t^{2}\right)^{-1 / 2}
$$

After logarithmic differentiation Eq. (1) becomes

$$
\frac{\mathrm{d} \rho_{\mathrm{f}}}{\rho_{\mathrm{f}}}-\frac{\mathrm{d} \rho_{0}}{\rho_{0}}=-\frac{\mathrm{d} f-\mathrm{d} A}{f(\alpha)-A}
$$

For $p=p_{0}$ and $r=r_{0}$ we deduce from Eq. (3) to (7):

$$
-(\mathrm{d} f-\mathrm{d} A)=\left[-\frac{\partial f}{\partial \alpha} \frac{\partial \alpha}{\partial k}+\frac{\partial A}{\partial k}+\frac{\partial A}{\partial H} \frac{\partial H}{\partial \alpha} \frac{\partial \alpha}{\partial k}\right] \mathrm{d} k
$$

or

$$
-(\mathrm{d} f-\mathrm{d} A)=U(k, p) . \mathrm{d} k
$$


If the variation in $k$ is due only to variation is $1_{0}$, equation (4) gives:

$$
\frac{\mathrm{d} k}{k}=-\frac{\mathrm{d} l_{0}}{l_{0}}
$$

Assuming $^{3}$ the Sommerfeld relation to be valid and the number of conduction electrons per unit volume to be temperature independent and assuming that the T.C.R. of bulk metallic layers is

$$
-\frac{\mathrm{d} l_{0}}{l_{0} \mathrm{~d} T}=\beta_{0}
$$

where $T$ is temperature Eq. (8) becomes:

$$
\beta_{\mathbf{f}}-\beta_{0}=\frac{k \cdot U(k, p)}{f(\alpha)-A} \cdot \beta_{0}
$$

or

$$
\beta_{\mathrm{f}} / \beta_{0}=\frac{1+k \cdot U(k, p)}{f(\alpha)-A}
$$

From eq. (1) we deduce:

$$
k \rho_{\mathrm{f}} / \rho_{0}=k[f(\alpha)-A]^{-1}
$$

By differentiating Eq. (14) we obtain

$$
\frac{\mathrm{d} k \rho_{\mathrm{f}} / \rho_{\mathbf{0}}}{k \rho_{\mathrm{f}} / \rho_{\mathbf{0}}}=\frac{\mathrm{d} k}{k}-\frac{\mathrm{d} f-\mathrm{d} A}{f(\alpha)-A}
$$

Substituting Eq. 9 in Eq. 14 we obtain

$$
\frac{\mathrm{d} k \rho_{\mathrm{f}} / \rho_{0}}{\mathrm{k} \rho_{\mathrm{f}} / \rho_{0}}=\left(1+\frac{k \cdot U(k, p)}{f(\alpha)}-\frac{\mathrm{d} k}{k}\right.
$$

Eq. (13) leads to

$$
\mathrm{d} k \rho_{\mathrm{f}} / \rho_{0}=\left(\rho_{\mathrm{f}} \beta_{\mathrm{f}}\right)\left(\rho_{0} \beta_{0}\right)^{-1} \mathrm{~d} k
$$

Assuming the Mathiessen's rule ${ }^{5}$ to be valid, we may write

$$
\rho_{\mathrm{f}}=\rho_{0}+\rho_{\mathrm{e}}
$$

where $\rho_{\mathrm{e}}$ is an extrinsic resistivity.

If $\rho_{\mathrm{e}}$ is essentially temperature independent, then

$$
\left(\rho_{\mathrm{f}} \beta_{\mathrm{f}}\right)\left(\rho_{0} \beta_{0}\right)^{-1}=1
$$

Since this assumption is not completely valid, we introduce the function $\mathrm{m}(r)$, measuring the deviation from the Mathiessen's rule, in the expression of $k \rho_{\mathrm{f}} / \rho_{0}$ related to a given value of $p$. Hence:

$$
\Delta\left[k \rho_{\mathrm{f}} / \rho_{0}\right]=[1+m(r)] \Delta k
$$

It may also be expressed as:

$$
k \rho_{\mathrm{f}} / \rho_{0}=\left[k \rho_{\mathrm{f}} / \rho_{0}\right]_{k=k_{0}}+[1+m(r)]\left[k-k_{0}\right]
$$

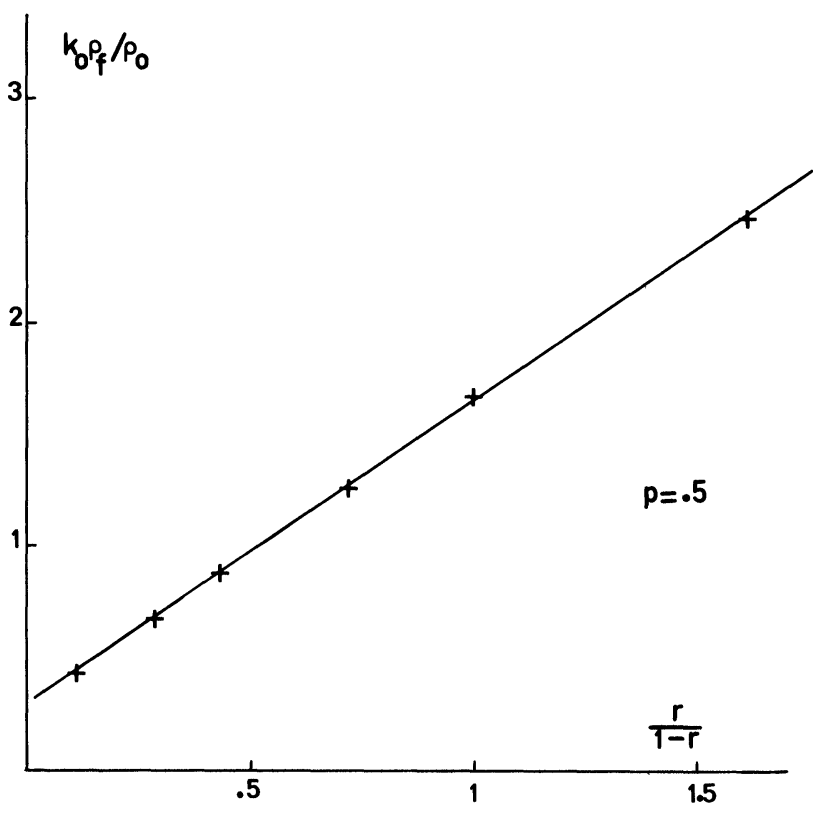

FIGURE 1 Exact values of $k_{0} \rho_{\mathrm{f}} / \rho_{0}$ versus $r /(1-r)$ for an electronic specular reflection coefficient, $p$, of 0.5 .

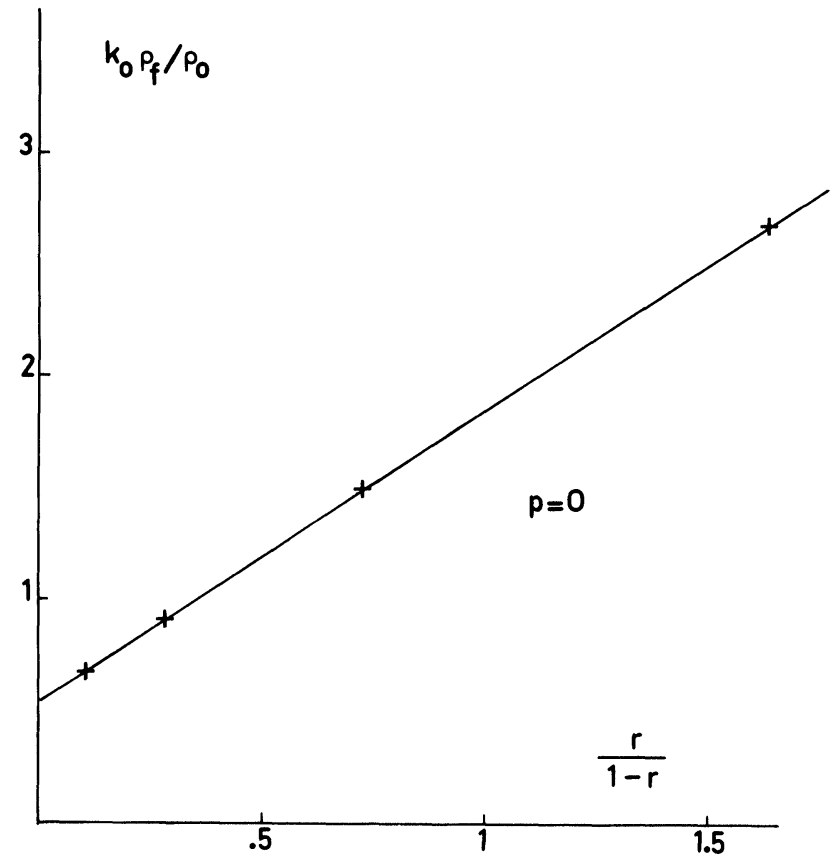

FIGURE 2 Exact values of $k_{0} \rho_{\mathrm{f}} / \rho_{0}$ versus $r /(1-r)$ for an electronic specular reflection coefficient of 0 . 
TABLE I

\begin{tabular}{llllll}
\hline & {$\left[k \rho_{\mathrm{f}} / \rho_{0}\right]_{k=0.1}$ Eq. (21) } & & \multicolumn{2}{l}{$\left[k \rho_{\mathrm{f}} / \rho_{0}\right]_{k=0.1}$ exact values } \\
\cline { 2 - 3 } \cline { 6 - 6 }$r$ & $p=0$ & $p=0.5$ & & $p=0$ & $p=0.5$ \\
\hline 0.1 & 0.676 & 0.4466 & & 0.6687 & 0.4336 \\
0.22 & 0.902 & 0.6723 & & 0.912 & 0.6685 \\
0.42 & 1.4858 & 1.2558 & & 1.4912 & 1.2579 \\
0.62 & 2.683 & 2.4536 & & 2.687 & 2.4613 \\
\hline
\end{tabular}

TABLE II

\begin{tabular}{|c|c|c|c|c|c|c|c|c|c|}
\hline \multirow[t]{2}{*}{$k$} & \multicolumn{3}{|c|}{ Thieme-Kirstein Eq. (24) } & \multicolumn{3}{|c|}{ Approx. Eq. (25) } & \multicolumn{3}{|c|}{$\mathrm{M}-\mathrm{S}$ exact values Eq. (1) } \\
\hline & $\begin{array}{l}p=0 \\
r=0,22\end{array}$ & $r=0.42$ & $r=0,62$ & $r=0,22$ & $r=0.42$ & $r=0.62$ & $r=0.22$ & $r=0.42$ & $r=0.62$ \\
\hline 0.05 & 0.8480 & 1.5112 & 2.8723 & 0.849 & 1.4345 & 2.632 & 0.8559 & 1.4392 & 2.6163 \\
\hline 0.1 & 0.8980 & 1.5612 & 2.9223 & 0.902 & 1.4858 & 2.683 & 0.912 & 1.4916 & 2.687 \\
\hline 0.5 & 1.2980 & 1.9612 & 3.3223 & 1.3256 & 1.8958 & 3.087 & 1.335 & 1.9058 & 3.0874 \\
\hline 1 & 1.7980 & 2.4612 & 3.8223 & 1.855 & 2.4083 & 3.592 & 1.8356 & 2.4137 & 3.6064 \\
\hline 2 & 2.798 & 3.4612 & 4.8223 & 2.914 & 3.4333 & 4.602 & 2.8268 & 3.4216 & 4.6348 \\
\hline$k$ & $\begin{array}{l}p=0.5 \\
r=0.22\end{array}$ & $r=0.42$ & $r=0.62$ & $r=0.22$ & $r=0.42$ & $r=0.62$ & $r=0.22$ & $r=0.42$ & $r=0.62$ \\
\hline 0.05 & 0.6605 & 1.3237 & 2.6848 & 0.6193 & 1.2045 & 2.4030 & 0.6141 & 1.2048 & 2.4084 \\
\hline 0.1 & 0.7105 & 1.3737 & 2.7348 & 0.6723 & 1.2558 & 2.4536 & 0.6685 & 1.2579 & 2.4613 \\
\hline 0.5 & 1.1105 & 1.7737 & 3.1348 & 1.0959 & 1.6658 & 2.8576 & 1.0879 & 1.6775 & 2.8836 \\
\hline 1 & 1.6105 & 2.2737 & 3.6348 & 1.6254 & 2.1783 & 3.3626 & 1.5967 & 2.1928 & 3.406 \\
\hline 2 & 2.6105 & 3.2737 & 4.6348 & 2.6844 & 3.2033 & 4.3726 & 2.6022 & 3.2106 & 4.4388 \\
\hline
\end{tabular}

TABLE III

\begin{tabular}{|c|c|c|c|c|c|c|c|c|}
\hline \multicolumn{5}{|c|}{$k \rho_{\mathrm{f}} / \rho_{0}$ Exact Eq. (1) } & \multicolumn{4}{|c|}{$k \rho_{\mathrm{f}} / \rho_{0}$ Approximate Eq. (25) } \\
\hline$k$ & $r=0.1$ & $r=0.22$ & $r=0.42$ & $r=0.62$ & $r=0.1$ & $r=0.22$ & $r=0.42$ & $r=0.62$ \\
\hline \multicolumn{9}{|l|}{$p=0$} \\
\hline 0.01 & 0.5487 & 0.8100 & 1.3973 & 2.5749 & 0.5779 & 0.8066 & 1.3935 & 2.592 \\
\hline 0.02 & 0.5631 & 0.8216 & 1.4079 & 2.5855 & 0.5888 & 0.8172 & 1.4038 & 2.602 \\
\hline 0.03 & 0.5769 & 0.8328 & 1.4178 & 2.5947 & 0.5997 & 0.8278 & 1.414 & 2.612 \\
\hline 0.04 & 0.5908 & 0.8444 & 1.4285 & 2.6056 & 0.6106 & 0.8384 & 1.4243 & 2.6224 \\
\hline 0.05 & 0.6043 & 0.8559 & 1.4392 & 2.6163 & 0.6215 & 0.8490 & 1.4345 & 2.632 \\
\hline 0.06 & 0.6175 & 0.8671 & 1.4494 & 2.6264 & 0.6324 & 0.8596 & 1.4448 & 2.6426 \\
\hline 0.07 & 0.6306 & 0.8785 & 1.4601 & 2.6371 & 0.6433 & 0.870 & 1.4550 & 2.6527 \\
\hline 0.08 & 0.6435 & 0.8897 & 1.4707 & 2.6478 & 0.6540 & 0.8808 & 1.4653 & 2.6628 \\
\hline 0.09 & 0.6561 & 0.9008 & 1.4810 & 2.6580 & 0.6650 & 0.8914 & 1.4755 & 2.6729 \\
\hline 0.1 & 0.6687 & 0.9120 & 1.4916 & 2.6870 & 0.6760 & 0.902 & 1.4858 & 2.683 \\
\hline 0.2 & 0.7873 & 1.0211 & 1.5960 & 2.7736 & 0.7850 & 1.0079 & 1.5883 & 2.784 \\
\hline 0.3 & 0.8941 & 1.1271 & 1.6997 & 2.8785 & 0.894 & 1.1138 & 1.6908 & 2.885 \\
\hline 0.4 & 1.0050 & 1.2310 & 1.8028 & 2.9831 & 1.003 & 1.2197 & 1.7933 & 2.986 \\
\hline 0.5 & 1.1090 & 1.3350 & 1.9053 & 3.0874 & 1.112 & 1.3256 & 1.8958 & 3.087 \\
\hline 0.6 & 1.2111 & 1.4350 & 1.0075 & 3.1916 & 1.221 & 1.4315 & 1.9983 & 3.188 \\
\hline 0.7 & 1.3120 & 1.5358 & 2.1093 & 3.2955 & 1.330 & 1.5374 & 2.1008 & 3.289 \\
\hline 0.8 & 1.4121 & 1.6360 & 2.2110 & 3.3993 & 1.439 & 1.6433 & 2.1928 & 3.390 \\
\hline 0.9 & 1.5116 & 1.7359 & 2.3124 & 3.5029 & 1.548 & 1.7492 & 2.3058 & 3.491 \\
\hline 1 & 1.6107 & 1.8356 & 2.4137 & 3.6064 & 1.657 & 1.855 & 2.4083 & 3.592 \\
\hline 2 & 2.5944 & 2.8268 & 3.4216 & 4.6348 & 2.747 & 2.914 & 3.4333 & 4.602 \\
\hline
\end{tabular}


We thus obtain approximate expressions of $k \rho_{\mathrm{f}} / \rho_{0}$ if the exact value of $\left[k \rho_{\mathrm{f}} / \rho_{0}\right]_{k=k_{0}}$ is known, slight deviations from the exact values (tabulated by Mola and $\mathrm{al}^{2}$ ) are obtained with $p$ independent values of $m(r)$.

Furthermore, we may observe that the exact values $^{2}$ of $k \rho_{\mathrm{f}} / \rho_{0}$ for $k=0.1$ are linear with $r / 1-r$ when $p=0$ or $p=0.5$ (Figure 1, 2). Hence:

$$
\begin{aligned}
\left(k \rho_{\mathrm{f}} / \rho_{0}\right)_{k=0.1} \approx & 0.1+\left[0.37(1-p)+0.06(1-p)^{2}\right] \\
& +1.32 \frac{r}{1-r}
\end{aligned}
$$

This expression is valid with a deviation less than $2 \%$ from the exact values in the 0 to $0.5 p$-range and in the 0.22 to $0.62 r$-range (Table I)

Making use of Mathiessen's rule Wissman ${ }^{6}$ had obtained

$$
\rho_{\mathrm{f}}=\rho_{0}\left[1+K \cdot \frac{1}{k}+\frac{3}{8} \cdot \frac{1-p}{k}\right]
$$

Thieme and Kirstein ${ }^{7}$ use

$$
K=\frac{3}{2} \cdot \frac{r}{1-r}
$$

Hence

$$
k \rho_{\mathrm{f}} / \rho_{0}=k+0.375(1-p)+1.5 \frac{r}{1-r}
$$

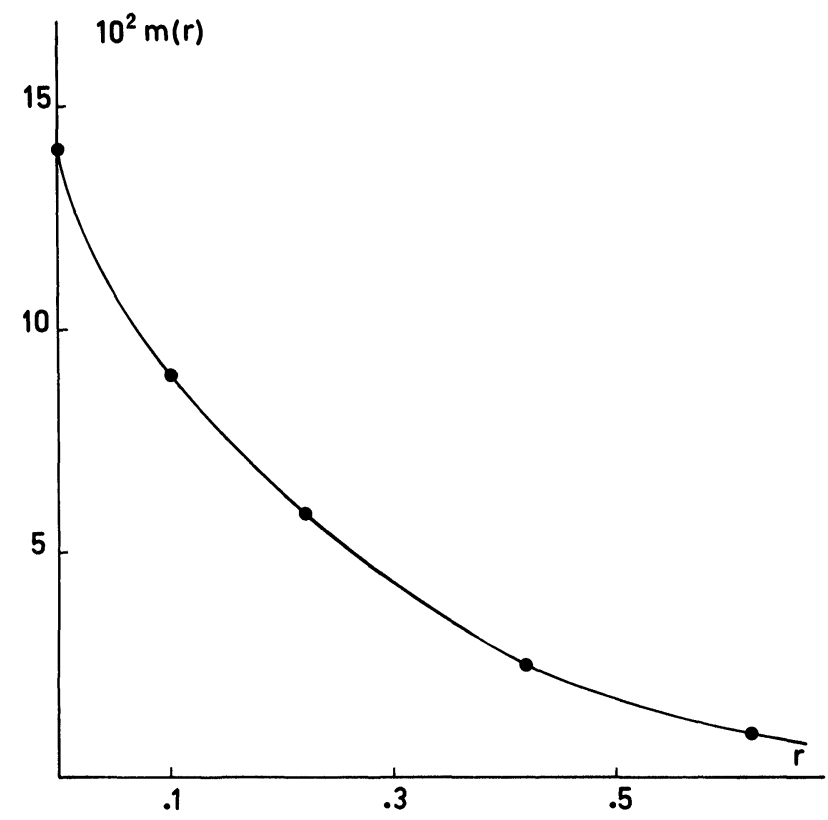

FIGURE 3 Variations in $m(r)$ (a measure of deviation from Mathiessen's rule) with $r$ (from Eq. 25).

In the ranges considered, this equation leads to most

\begin{tabular}{|c|c|c|c|c|c|c|c|c|}
\hline \multirow[b]{2}{*}{$k$} & \multicolumn{4}{|c|}{$k \rho_{\mathrm{f}} / \rho_{0}$ Exact Eq. (1) } & \multicolumn{4}{|c|}{$k \rho_{\mathrm{f}} / \rho_{0}$ Approximate Eq. (25) } \\
\hline & $r=0.1$ & $r=0.22$ & $r=0.42$ & $r=0.62$ & $r=0.1$ & $r=0.22$ & $r=0.42$ & $r=0.62$ \\
\hline \multicolumn{9}{|c|}{$p=0.5$} \\
\hline 0.01 & 0.3280 & 0.5700 & 1.1624 & 2.3665 & 0.3485 & 0.5769 & 1.1635 & 2.3627 \\
\hline 0.02 & 0.3403 & 0.5811 & 1.1731 & 2.3772 & 0.3594 & 0.5875 & 1.1738 & 2.3728 \\
\hline 0.03 & 0.3522 & 0.5919 & 1.1832 & 2.3866 & 0.3703 & 0.5981 & 1.8140 & 2.3829 \\
\hline 0.04 & 0.3642 & 0.6030 & 1.1941 & 2.3976 & 0.3812 & 0.6087 & 1.1943 & 2.3930 \\
\hline 0.05 & 0.3761 & 0.6141 & 1.2048 & 2.4084 & 0.3921 & 0.6193 & 1.2045 & 2.4030 \\
\hline 0.06 & 0.3877 & 0.6249 & 1.2153 & 2.4186 & 0.4030 & 0.6299 & 1.2148 & 2.4132 \\
\hline 0.07 & 0.3993 & 0.6359 & 1.2260 & 2.4294 & 0.4139 & 0.6405 & 1.2250 & 2.4233 \\
\hline 0.08 & 0.4109 & 0.6469 & 1.2367 & 2.4402 & 0.4248 & 0.6511 & 1.2353 & 2.4334 \\
\hline 0.09 & 0.4222 & 0.6577 & 1.2472 & 2.4505 & 0.4357 & 0.6617 & 1.2455 & 2.4435 \\
\hline 0.1 & 0.4336 & 0.6685 & 1.2579 & 2.4613 & 0.4466 & 0.6723 & 1.2558 & 2.4536 \\
\hline 0.2 & 0.5435 & 0.7755 & 1.3638 & 2.5672 & 0.5556 & 0.7782 & 1.3583 & 2.5546 \\
\hline 0.3 & 0.6497 & 0.8808 & 1.4689 & 2.673 & 0.6646 & 0.8841 & 1.4608 & 2.6556 \\
\hline 0.4 & 0.7538 & 0.9847 & 1.5735 & 2.7785 & 0.7736 & 0.9900 & 1.5633 & 2.7566 \\
\hline 0.5 & 0.8566 & 1.0879 & 1.6775 & 2.8836 & 0.8826 & 1.0959 & 1.6658 & 2.8576 \\
\hline 0.6 & 0.9586 & 1.1904 & 1.7812 & 2.9886 & 0.9916 & 1.2018 & 1.7683 & 2.9586 \\
\hline 0.7 & 1.0600 & 1.2924 & 1.8845 & 3.0932 & 1.1006 & 1.3077 & 1.8708 & 3.0596 \\
\hline 0.8 & 1.1610 & 1.3941 & 1.9875 & 3.1976 & 1.209 & 1.4136 & 1.9733 & 3.1606 \\
\hline 0.9 & 1.2618 & 1.4955 & 2.0903 & 3.3019 & 1.3186 & 1.5195 & 2.0758 & 3.2616 \\
\hline 1 & 1.3622 & 1.5967 & 2.1928 & 3.4060 & 1.4276 & 1.6254 & 2.1783 & 3.3626 \\
\hline 2 & 2.3622 & 2.6022 & 3.2106 & 4.4388 & 2.5176 & 2.6844 & 3.2033 & 4.3726 \\
\hline
\end{tabular}
significant deviations (Table II).

Values of $\left(k \rho_{\mathrm{f}} / \rho_{0}\right)$ calculated from Eq. (21) are reported in Eq. (20). Hence

TABLE IV 


$$
\begin{aligned}
k \rho_{\mathrm{f}} / \rho_{0} \approx & 0.1+\left[0.37(1-p)+0.06(1-p)^{2}\right] \\
& +1.32 \cdot \frac{r}{1-r}+[1+m(r)][k-0.1]
\end{aligned}
$$

Variation of $m(r)$ with $r$ is given in Fig. 3.

Deviation of less than $6 \%$ is obtained in the 0.01 to $2 k$-range, 0 to $0.5 p$-range, 0.1 to $0.62 r$-range.

(Tables III and IV).

\section{CONCLUSION}

Under the assumption that the effects due to surface scattering and to grain-boundary scattering may be treated separately, we obtain an approximate expression containing a term directly related to grainboundary scattering and another related to surface scattering. A correction term is necessary for a complete description of surface scattering. We also must introduce a term describing the combined action of isotropic scattering and grain scattering. We shall attempt to give further theoretical considerations to this point in a future paper.

\section{REFERENCES}

1. A. F. Mayadas and M. Shatzkes, Phys. Rev. B, 1, 1382-1389 (1970).

2. E. E. Mola and J. M. Heras, Electrocomp. Sci. Tech., 1, 77-80 (1974).

3. E. E. Mola and J. M. Heras, Thin Solid Films, 18, 137-144 (1973).

4. T. J. Coutts, Thin Solid Films, 7, 77-100 (1971).

5. L. I. Maissel in "Hand book of Thin Film Technology", McGraw-Hill Book Co., (1970) p. 13-4.

6. P. Wissman, Thin Solid Films, 5, 329-338 (1970).

7. F. Thieme and W. Kirstein, Thin Solid Films, 30, 371-375 (1975). 

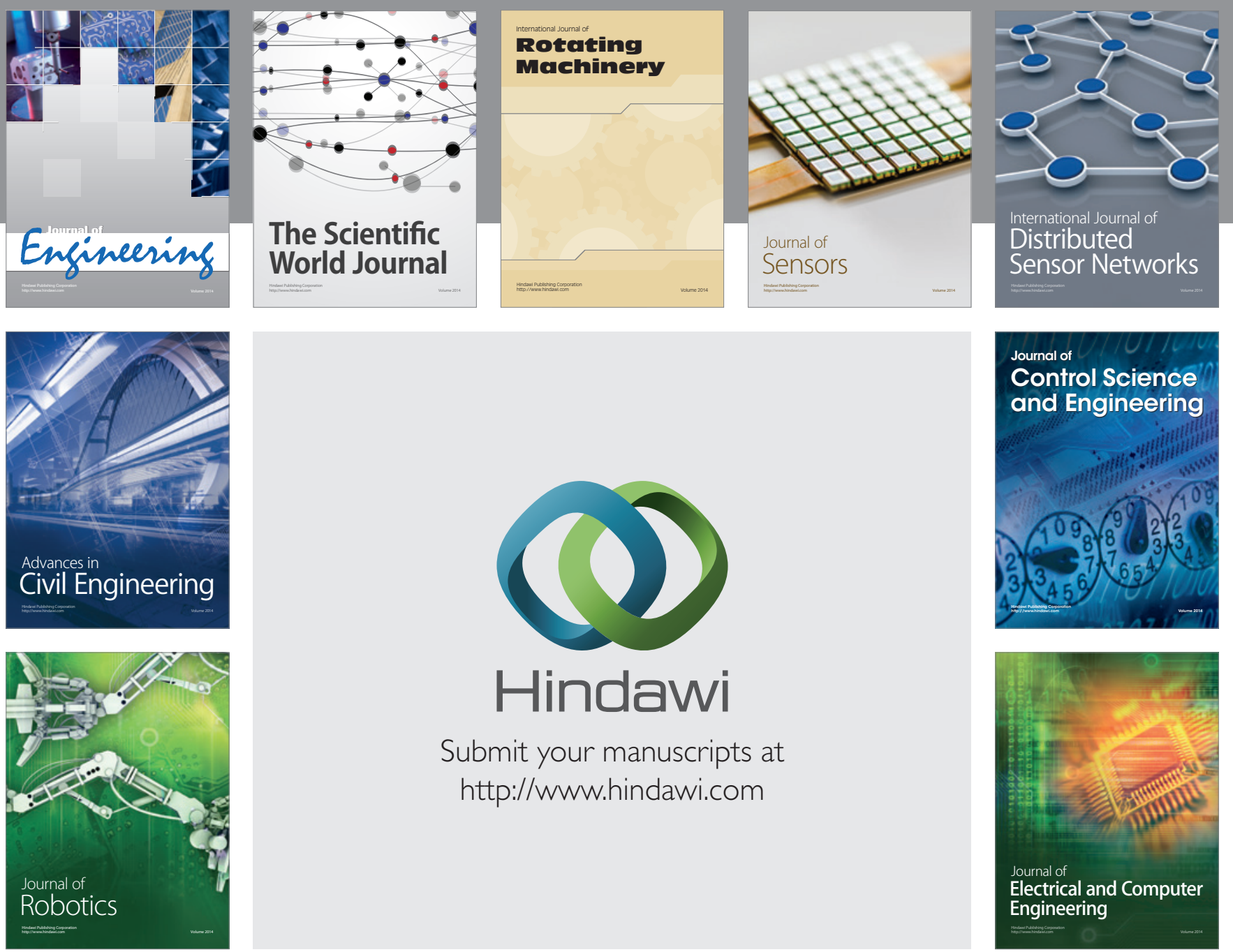

Submit your manuscripts at

http://www.hindawi.com
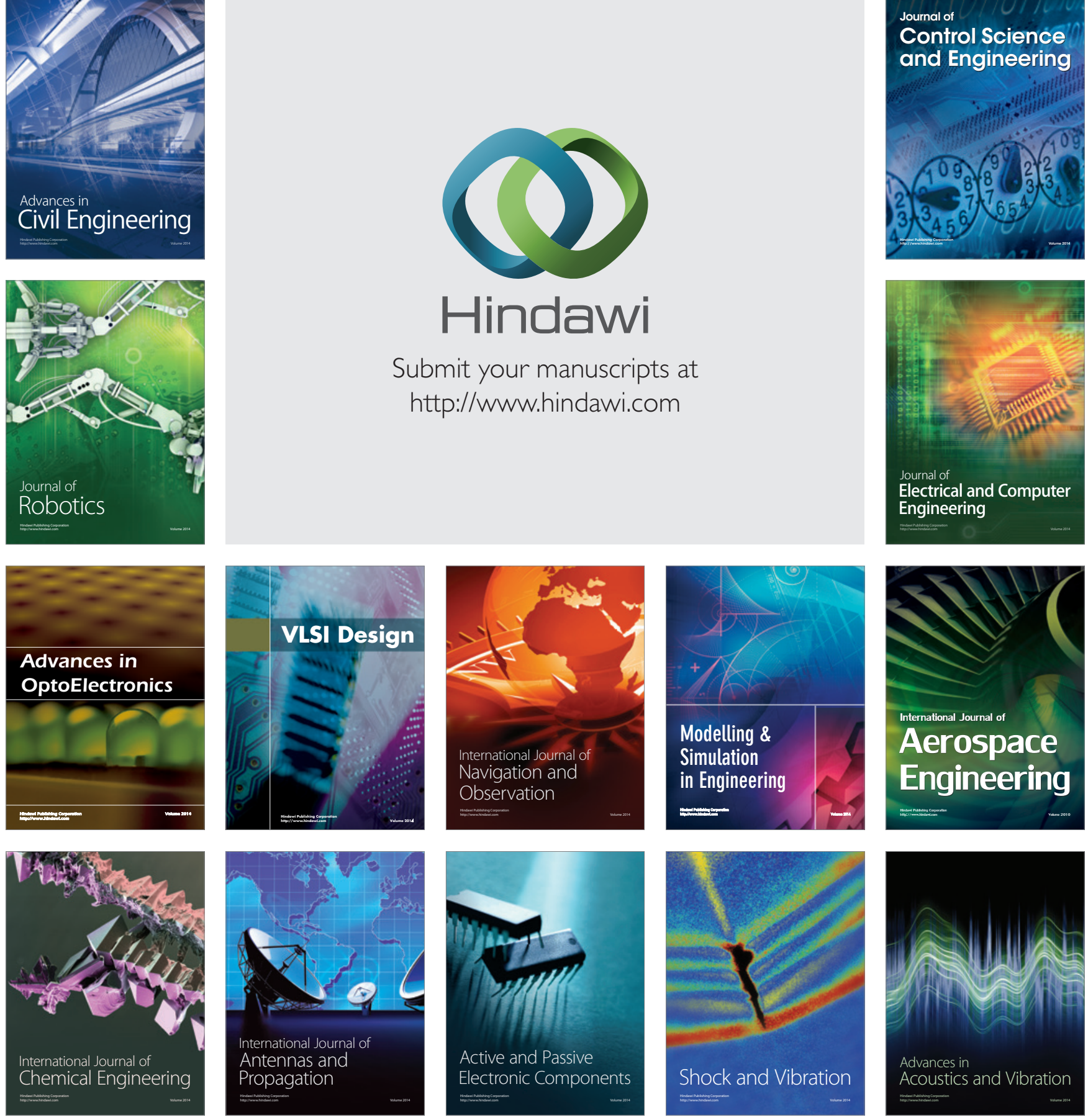